\title{
Symptom Dimensions and Clinical Presentations in Obsessive Compulsive Disorder
}

\author{
Hemant $\operatorname{Kumar}^{1} *$
}

\section{ABSTRACT}

In Obsessive compulsive disorder (OCD) a wide range of symptoms are seen, along with different responses to treatment, this has pointed to the direction that OCD is more heterogeneous than thought before and clinically different subtypes of OCD exist. This article presents a wide variety of symptoms dimension seen in OCD with many pioneering and important work done in this area. The different clinical presentations of patients with OCD have also been seen. The heterogeneity and the vastness of the symptoms of this disorder could lead to new breakthroughs and pathways in our current understanding of OCD.

Keywords: Obsessive Compulsive Disorder (OCD), Heterogeneity

Obsessive compulsive disorder is a distressing and debilitating Psychiatric disorder effecting from $1 \%$ to $3 \%$ of the general population (Weissman, Leaf et al. 1988). The symptoms presented in OCD are very different from those which may be found in other anxiety disorders, each patient could report a different range of symptoms, which is often indicative a the vastness of this disorder. However, it is seen that there is a consistency in symptoms across time and culture (Clark 2004).. In turn, it became of increasing importance that these claims be put to test and investigation of the occurrence of these subtypes is, and so several of these methods have been created to investigate the differences in treatments and the etiology of the disorder. The overt symptoms are primarily used to identify the subtype dimensions which currently exists (i.e., classifying patients as washers, checkers, hoarders, etc.) for sub typing schemes (McKay, Abramowitz et al. 2004). Since the earliest times, attempts have been made by the researchers to dissect the phenotype into homogeneous subtypes. In a study pioneering study Falrethad drawn out the distinction between folie du doute ("madness of doubt") and délire du toucher ("delusion of touch") in 1869 (4). Investigators have often distinguished the "washers" from "checkers" (5) and other symptom-based clusters (6-8). Classification of patients into extremes of a continum by authors has also been done, for example, impulsivity (9) or insight $(10,11)$. Generally, these

\footnotetext{
${ }^{1}$ Research Scholar, Patna University, At. Mansi Araiya Dist.Khagaria, Bihar India

*Responding Author

(C) 2016 I H Kumar; licensee IJIP. This is an Open Access Research distributed under the terms of the Creative Commons Attribution License (http://creativecommons.org/licenses/by/2.0), which permits unrestricted use, distribution, and reproduction in any Medium, provided the original work is properly cited.
} 


\section{Symptom Dimensions and Clinical Presentations In Obsessive Compulsive Disorder}

attempts had limited success in relating the identified subtypes to biological markers, genetic factors, or treatment response, in part because pure subtypes of patients are rare and the recruitment of sufficient sample sizes of each subtype is difficult and highly impractical. Other putative subtypes have been identified based on clinical characteristics, such as age at onset (12) and co morbid diagnoses, particularly tic disorders (13). Limitations of these approaches include knowing exactly when the obsessive-compulsive symptoms began and the difficulty of identifying "hidden" tic-related cases (individuals who have relatives with tic disorders but no tics of their own).

\section{THE SYMPTOM DIMENSIONS}

The Yale -Brown Obsessive-Compulsive Scale-Symptom Checklist was first factor analyzed (YBOCS-SC) (Goodman, Rasmussen et al. 1995) (Baer 1994). The 13 main categories in YBOCS -SC were factor analyzed, 107 patients comprised of the sample and three factors were identified, the variance was found to be $48 \%$ among the factors; these were named "symmetry/ hoarding," "contamination/ cleaning," and "pure obsessions". After this influential work by Baer, Leckmanalong with his colleagues (Leckman, Grice et al. 1997) again assessed the same 13 categories which are used to group types of obsessions and compulsions in the YBOCS-SC in two large groups of OCD , with over 300sample size(Pauls, Towbin et al. 1986, Leckman, Walker et al. 1994). To identify valid "traits," any Symptoms which occur in OCD among ever in their experience over the span of their lifetimes would be taken; this was in contrast to taking only current symptoms into account. As a surprise, both of these researches' brought out identical results. Four factors were identified that in total accounted for over $60 \%$ of the variance in each data set (Leckman, Grice et al. 1997). Subsequently, Summerfeldt and colleagues (Summerfeldt, Richter et al. 1999) evaluated existing models of OCD symptom structure in 203 individuals. Confirmatory fact analysis was used; they determined that, there was an "adequate fit" solely for a four factor model. A more recent meta-analysis was done, in which the data from 21 studies comprising of 5124 participants was studied, this study established the same four factors as valid(Bloch, Landeros-Weisenberger et al. 2008). Studies were examined whether they involved participants with OCD and included an exploratory factor analysis of the 13 YBOCS-SC categories and the items therein. (Goodman, Rasmussen et al. 1995) Stratified metaanalysis was done to settle on the factor structure of OCD in studies involving children and adults separately. The four factors were: (Factor I) Forbidden thoughts - aggression, sexual, religious, and somatic obsessions and checking compulsions; (Factor II) Symmetry - symmetry obsessions and repeating, ordering, and counting compulsions; (Factor III) Cleaning - cleaning and contamination; and (Factor IV) Hoarding - hoarding obsessions and compulsions. In a Factor analysis of studies done including on adults, it yielded a factor structure which was identical compared with the overall meta-analysis. The only differences between the factor structures involving adults and children were: (i) checking compulsions loaded highest on the Forbidden thoughts factor in adults and with the Symmetry factor in children; and (ii) somatic obsessions were highest on the Forbidden thoughts factor in adults and with the Cleaning factor in children. 


\section{Symptom Dimensions and Clinical Presentations In Obsessive Compulsive Disorder}

The shifting of checking symptoms from one factor to another could be attributed to the natural ambiguity of checking symptoms in the Y-BOCS-SC. This ambiguity in the checking category of the Y-BOCS-SC has been addressed in the dimensional OCD scales such as the Dimensional Yale-Brown Obsessive Compulsive Scale (DY-BOCS), which links specific checking and avoidance OC symptoms with each OC symptom dimension/ factor (Rosario-Campos, Miguel et al. 2006).

Although this dimensional structure understanding of OC symptoms is still far from perfect, this quantitative approach to phenotypic traits has the potential to press forward the understanding of OCD, and may assist in the identification of more strong endopheno types.(Leckman, Bloch et al. 2009).

\section{CLINICAL PRESENTATIONS AND SUBTYPES OF OCD}

The clinical presentation and subtypes of the OCD symptom-based subtypes identified in previous studies is explained.(Abramowitz, Franklin et al. 2003, Calamari, Wiegartz et al. 2004) contamination/ washing, harm obsessions/checking, pure obsessions, and hoarding.

\section{Contamination and Washing/Cleaning}

Feinstein et al, 2003 (Feinstein, Fallon et al. 2003) found evidence of two different groups of OCD patients with repetitive washing: (a) those who report feeling of discomfort or contamination without any fears of harm; and (b) those with definite fears of harm as a result of perceived contamination. Patients in the first type usually focus on the feeling of contamination and report fewer obsessions. Washing and cleaning excessively is done so that the feeling of contamination reduces. The second types of patients are more focused on the frightening consequences of contamination, including responsibility for spreading contaminants to others. Washing or checking rituals among such individuals are performed to avert perceived danger.

\section{Harm Obsessions and Checking Rituals}

Most variability is shown in Aggressive obsessions and checking compulsions in how they cluster in factor analytic studies (Feinstein, Fallon et al. 2003) and the range of Obsession content and related checking behaviour demonstrates the vast heterogeneity in OCD, even within this subtype. Checkers may report intrusive thoughts of harm which they think may increase the likelihood of specific feared events happening, and in turn may perform particular rituals to prevent occurrence to self or others perceived as vulnerable, (Rachman 1997, Sookman and Pinard 2002). Patients with sexual or aggressive thoughts and images may check to relieve doubt as to their actual dangerous behaviour. Neutralizing responses carried out to decrease anxiety about intrusions paradoxically increase the frequency of intrusions (Salkovskis, Wroe et al. 2000). Some patients often exaggerate the magnitude of mistakes, or they overestimate their responsibility for potential disasters and therefore check to ensure safety. Cognitive biases such as thought action fusion are often seen in checkers than in washers. (Shafran, Thordarson et al. 


\section{Symptom Dimensions and Clinical Presentations In Obsessive Compulsive Disorder}

1996) e.g., “a bad thought is as bad as an action”') leading to the terrible misappraisal of naturally occurring intrusions as dangerous.

\section{Obsessions without Overt Compulsions}

$25 \%$ of OCD patients report obsessions which are troubling without overt compulsions. Common themes in obsessions are sex, harm/violence, and religion/blasphemy. As of not long ago, this subgroup was viewed as impervious to CBT and medication (Griest, 1990). Patients who had these manifestations often recognise their distressing thoughts to be dangerous, overly important, and hence they try to control such thoughts (Obsessive Compulsive Cognitions Working Group, 2001). Mental rituals and neutralizing are often carried out to decrease anxiety associated with intrusions when they are experienced as involuntary and overwhelming. These responses appear to be functionally similar to overt compulsions (Salkovskis 1985).

\section{Segregation of Hoarding from Obsessive Compulsive Symptoms}

Hoarding was thought to be OC symptoms but the phenotypes of both hoarding and OC symptoms may be etiologically and clinically distinct as suggested by the recent evidences. Hoarding has been kept as a new diagnosis in DSM V, though previously it was kept as a subtype in OCD but multiple evidences has suggested that pathological hoarding may be etiologically distinct (Pertusa, Fullana et al. 2008, Mataix-Cols, Frost et al. 2010). Taylor et al. 2010 found that in 167 monozygotic and 140 dizygotic twins both male and female had 42\% of the phenotype variance of hoarding which attributed to the genetic factors. C. A. Mathews et al., 2013 suggested that in the etiology of both hoarding and OC symptoms there is a contribution from that of the independent genetic factors where the heritability were estimated to 0.36 for hoarding and 0.40 for OC symptoms. In the previous studies the heritability estimate was found to be lower $40-50 \%$ for OC symptoms and $35-50 \%$ for OC symptoms (Van Grootheest, Bartels et al. 2008, Taylor, Jang et al. 2010). It has been suggested that three of the hoarding behaviours happen owing to environmental factors and the role of the environmental factors has been evidenced by the twin studies instead of genetic studies. Data have been found to be unreliable concerning trauma and abuse in the progress of hoarding and OC symptoms (Taylor, Jang et al. 2010) and in understanding the etiology in the progression of the hoarding and OC symptoms in accord to the genetic and environmental factors has found to be the next essential step.

\section{CONCLUSION}

Variations among OCD symptom dimensions are more varied than as thought earlier. In recent time more and more studies have forced even DSM-V to consider adding more specification to this disorder. Through the years OCD as seen a narrow down approach in terms of studying various aspects involved in it. It will not be matter of surprise to expect more nosological issues and diagnostic entities emerging out of this fascinating disorder. 


\section{Symptom Dimensions and Clinical Presentations In Obsessive Compulsive Disorder}

\section{Acknowledgments}

The author appreciates all those who participated in the study and helped to facilitate the research process.

\section{Conflict of Interests}

The author declared no conflict of interests.

\section{REFERENCE}

Abramowitz, J. S., M. E. Franklin, S. A. Schwartz and J. M. Furr (2003)."Symptom presentation and outcome of cognitive-behavioral therapy for obsessive-compulsive disorder."Journal of Consulting and Clinical Psychology 71(6): 1049.

Baer, L. (1994). "Factor analysis of symptom subtypes of obsessive compulsive disorder and their relation to personality and tic disorders. "Journal of Clinical Psychiatry.

Bloch, M., A. Landeros-Weisenberger, M. Rosario, C. Pittenger and J. Leckman (2008)."Metaanalysis of the symptom structure of obsessive-compulsive disorder." American Journal of Psychiatry 165(12): 1532-1542

Calamari, J. E., P. S. Wiegartz, B. C. Riemann, R. J. Cohen, A. Greer, D. M. Jacobi, S. C. Jahn and C. Carmin (2004). "Obsessive-compulsive disorder subtypes: an attempted replication and extension of a symptom-based taxonomy." Behavior Research and Therapy 42(6): 647-670.

Calamari, J. E., P. S. Wiegartz, B. C. Riemann, R. J. Cohen, A. Greer, D. M. Jacobi, S. C. Jahn and C. Carmin (2004). "Obsessive-compulsive disorder subtypes: an attempted replication and extension of a symptom-based taxonomy." Behavior Research and Therapy 42(6): 647-670.

Clark, D. A. (2004).Cognitive-behavioral therapy for OCD, Guilford Press.

Feinstein, S. B., B. A. Fallon, E. Petkova and M. R. Liebowitz (2003)."Item-by-item factor analysis of the Yale-Brown Obsessive Compulsive scale symptom checklist." The Journal of Neuropsychiatry and Clinical Neurosciences 15(2): 187-193.

Goodman, W., S. Rasmussen and J. F. Leckman (1995). "A family study of obsessive-compulsive disorder." American Journal of Psychiatry 152: 76-84.

Goodman, W., S. Rasmussen and J. F. Leckman (1995)."A family study of obsessive-compulsive disorder." American Journal of Psychiatry 152: 76-84.

Greist, J. H., B. Bandelow, E. Hollander, D. Marazziti, S. A. Montgomery, D. J. Nutt, A. Okasha, R. P. Swinson and J. Zohar (2003). "WCA recommendations for the long-term treatment of obsessive-compulsive disorder in adults." CNS Spectrum 8(suppl 1): 7-16.

Leckman, J. F., D. E. Walker, W. K. Goodman, D. L. Pauls and D. J. Cohen (1994)."" Just Right" perceptions associated with compulsive behavior in Tourette's syndrome." American Journal of Psychiatry 151(5): 675-680.

McKay, D., J. S. Abramowitz, J. E. Calamari, M. Kyrios, A. Radomsky, D. Sookman, S. Taylor and S. Wilhelm (2004). "A critical evaluation of obsessive-compulsive disorder subtypes: symptoms versus mechanisms." Clinical Psychology Review 24(3): 283-313.

Pauls, D. L., K. E. Towbin, J. F. Leckman, G. E. Zahner and D. J. Cohen (1986). "Gilles de la Tourette's syndrome and obsessive-compulsive disorder: evidence supporting a genetic relationship." Archives of General Psychiatry 43(12): 1180-1182. 


\section{Symptom Dimensions and Clinical Presentations In Obsessive Compulsive Disorder}

Pertusa, A., M. Fullana, S. Singh, P. Alonso, J. Menchón and D. Mataix-Cols (2008). "Compulsive hoarding: OCD symptom, distinct clinical syndrome, or both?" American Journal of Psychiatry 165(10): 1289-1298.

Rachman, S., (1997)."A cognitive theory of obsessions." Behavior Research and Therapy 35(9): 793-802.

Rasmussen, S.A., (1994)."Obsessive compulsive spectrum disorders."Journal of Clinical Psychiatry.

Rosario-Campos, M. d., E. Miguel, S. Quatrano, P. Chacon, Y. Ferrao, D. Findley, L. Katsovich, L. Scahill, R. King and S. Woody (2006). "The Dimensional Yale-Brown ObsessiveCompulsive Scale (DY-BOCS): an instrument for assessing obsessive-compulsive symptom dimensions." Molecular Psychiatry 11(5): 495-504.

Rosario-Campos, M. d., E. Miguel, S. Quatrano, P. Chacon, Y. Ferrao, D. Findley, L. Katsovich, L. Scahill, R. King and S. Woody (2006). "The Dimensional Yale-Brown ObsessiveCompulsive Scale (DY-BOCS): an instrument for assessing obsessive-compulsive symptom dimensions." Molecular Psychiatry 11(5): 495-504.

Salkovskis, P. M. (1985). "Obsessional-compulsive problems: A cognitive-behavioural analysis." Behavior Research and Therapy 23(5): 571-583.

Salkovskis, P. M., A. Wroe, A. Gledhill, N. Morrison, E. Forrester, C. Richards, M. Reynolds and S. Thorpe (2000). "Responsibility attitudes and interpretations are characteristic of obsessive compulsive disorder." Behavior Research and Therapy 38(4): 347-372.

Shafran, R., D. S. Thordarson and S. Rachman (1996)."Thought-action fusion in obsessive compulsive disorder. "Journal of Anxiety Disorders 10(5): 379-391.

Sookman, D. and G. Pinard (2002)."Overestimation of threat and intolerance of uncertainty in obsessive compulsive disorder. "Cognitive Approaches to Obsessions and Compulsions: Theory, Assessment and Treatment: 63-89.

Summerfeldt, L. J., M. A. Richter, M. M. Antony and R. P. Swinson (1999). "Symptom structure in obsessive-compulsive disorder: a confirmatory factor-analytic study." Behavior Research and Therapy 37(4): 297-311.

Taylor, S., K. L. Jang and G. J. Asmundson (2010)."Etiology of obsessions and compulsions: $a$ behavioral-genetic analysis."Journal of Abnormal Psychology 119(4): 672.

Weissman, M. M., P. J. Leaf, G. L. Tischler, D. G. Blazer, M. Karno, M. L. Bruce and L. P. Florio (1988). "Affective disorders in five United States communities. "Psychological Medicine 18(01): 141-153.

How to cite this article: H Kumar (2016), Symptom Dimensions and Clinical Presentations In Obsessive Compulsive Disorder, International Journal of Indian Psychology, Volume 3, Issue 4, No. 59, ISSN 2348-5396 (e), ISSN: 2349-3429 (p), DIP: 18.01.069/20160304, ISBN: 978-1-36526307-1 